\title{
Banco Mundial de Datos sobre Salud Bucodental de la OMS, 1986-1996: panorámica de las encuestas de salud bucodental a los 12 años de edad'1
}

\author{
A. Nithila, ${ }^{2}$ D. Bourgeois, ${ }^{2}$ D. E. Barmes ${ }^{3}$ y H. Murtomaa ${ }^{4}$
}

RESUMEN En el presente artículo se describe la situación mundial de la salud bucodental de los niños de 12 años de edad —el índice de dientes cariados, perdidos y obturados (CPO) y el porcentaje de la población afectada- a partir de los estudios representativos más recientes sobre 80 países incluidos en el Banco Mundial de Datos sobre Salud Bucodental (BMDSB) de la OMS entre 1986 y 1996.

El volumen de información varió mucho: $68 \%$ de las economías de mercado de los países desarrollados tenían por lo menos un conjunto nacional de datos, en comparación con $38 \%$ de las economías de los países en desarrollo y 36\% de las economías en transición. Las proporciones en cada Región de la OMS fueron las siguientes: Mediterráneo Oriental, 55\%; Europa, 50\%; Pacífico Occidental, 48\%; África, 39\%; Asia Sudoriental, 30\%; y las Américas, 26\%.

En el mundo en general, el índice ponderado de dientes CPO en todos los datos del BMDSB es $<3,0 \%$, que es la meta de la OMS/Federación Dental Internacional para el año 2000. Con respecto a los datos reseñados en el presente artículo, se discuten el logro y el incumplimiento de esa meta, al igual que la variación del indice medio de dientes CPO y la proporción de niños afectados en varias agrupaciones de países. Hay dificultades para obtener datos recientes sobre muchos países, pero en el artículo se recalca la necesidad de mantener y ampliar el BMDSB para facilitar la recopilación de datos de salud bucodental válidos, fidedignos y comparables.

El Banco Mundial de Datos sobre Salud Bucodental (BMDSB) se estableció en 1969 para atender a la necesidad

\footnotetext{
1 Se publicó en inglés en el Bulletin of the World Health Organization, Vol. 76, No. 3, 1998, con el título "WHO Global Oral Data Bank, 1986-96: An overview of oral health surveys at 12 years of age". c) Organización Mundial de la Salud, 1998.

2 Organización Mundial de la Salud, División de Enfermedades no Transmisibles, Programa de Salud Bucodental, Ginebra, Suiza.

3 Instituto Nacional de Investigación Dental, Institutos Nacionales de Salud, Bethesda, MD, E.U.A. Las solicitudes de separatas deben enviarse a este autor.

4 Universidad de Helsinki, Departamento de Salud Pública Dental, Helsinki, Finlandia.
}

de información sobre el estado de la salud bucodental y las tendencias epidemiológicas, que sirviera de base para el Programa de Salud Bucodental de la OMS (ORH). En la actualidad, el BMDSB contiene 1850 conjuntos de datos sobre caries dental en 178 países. Con el transcurso de los años, se ha convertido en la principal fuente de referencia internacional sobre la epidemiología mundial de la salud bucodental. Los datos sobre salud bucodental se emplean de varias formas: para evaluar la situación nacional, regional y mundial, orientar a los pro- gramas y realizar actividades de planificación y vigilancia (1).

Cada conjunto de datos es verificado por la OMS para determinar que se ciña a criterios y metodología uniformes antes de ser aceptado para su incorporación al BMDSB. De esa forma, el banco de datos atiende a las solicitudes de recopilación de información de salud bucodental claramente definida y disponible en el ámbito internacional (2) y la exigencia de normalización del sistema de acopio y presentación de esos datos (3). Además, el BMDSB acumula información pertinente de fuen- 
tes que no suelen figurar en las publicaciones científicas, por ejemplo, los informes preparados para los ministerios de salud. La OMS también recomienda que los países realicen encuestas periódicas de salud bucodental cada cinco años si es posible, con el fin de captar los cambios de los patrones y tendencias de la salud bucodental suficientemente pronto para realizar una planificación o replanificación de los servicios de forma apropiada y oportuna.

En el presente boletín de actualidades se examinan los datos sobre la caries dental disponibles en el BMDSB, acopiados en el período 1986-1996 para la edad índice de 12 años (1). Se escogieron solamente las encuestas nacionales $\mathrm{y}$ aquellas encuestas subnacionales a partir de las cuales se pudieran hacer estimaciones nacionales aceptables.

\section{MATERIALES Y MÉTODOS}

La información del BMDSB se acopia de las fuentes citadas a continuación:

- informes de encuestas hechas con los métodos epidemiológicos normales de la OMS, incluso con metodología exploratoria, como se describe en Oral health surveys: Basic methods (4);

- artículos sobre salud bucodental de la base de datos MEDLINE, siempre y cuando se ciñan a los criterios y la metodología establecidos por la OMS y puedan resumirse en el formato estándar; e

- informes oficiales de las oficinas regionales de la OMS, los ministerios de salud y las instituciones de investigación que usan los métodos normales de la OMS.

En el período 1986-1996 se incluyó en el BMDSB un total de 307 encuestas de niños de 12 años. Se clasificaron como encuestas nacionales, regionales o locales, o con información limitada solamente. Hemos incluido aquí las siguientes clases de datos de todo el conjunto:

- datos nacionales obtenidos por medio de muestreo aleatorio, estudios exploratorios o registros nacionales y
- datos regionales considerados adecuados por las autoridades locales y la OMS como base de estimaciones nacionales fidedignas.

Cuando hubo más de una encuesta que se ciñera a esos criterios en algún país, se citó aquí solamente la última.

Los datos presentados incluyen el porcentaje de niños afectados en la muestra, el índice de dientes cariados, perdidos y obturados (CPO) $\mathrm{y}$, si existe la información, los componentes de dientes cariados $(\mathrm{C})$, perdidos $(\mathrm{P})$ y obturados (O). Para la presentación de los datos se han empleado dos clasificaciones de los Estados Miembros. Primero, por región de la OMS: África, las Américas, el Mediterráneo Oriental, Europa, Asia Sudoriental y el Pacífico Occidental, y segundo, de conformidad con los criterios citados en World economic and social survey 1994 (5), en que los países se clasifican como economías de mercado en desarrollo, desarrolladas o en transición.

\section{RESULTADOS}

\section{Encuestas representativas de salud bucodental a los 12 años de edad en el BMDSB}

Aquí se informa sobre 80 de las 307 encuestas incluidas en el BMDSB en el período 1986-1996 y se proporcionan datos sobre $42 \%$ de los Estados Miembros de la OMS. En un principio, los Estados Miembros que forman parte del BMDSB se subdividieron sencillamente en países en desarrollo o muy industrializados, clasificación que destacaba marcados contrastes en la prevalencia de la caries dental y sus tendencias. La clasificación económica más reciente en tres categorías ha acentuado más esos contrastes. Al subdividir los datos en categorías de países, las 17 encuestas de las economías de mercado de los países desarrollados representan $68 \%$ de esa categoría y los datos de los ocho países con economías en transición representan 36\% de esa categoría. Sin subdividir los países en desarrollo en categorías de países menos desarrollados y otros, las 55 encuestas de los países en desarrollo representan $38 \%$ de esa categoría. La fig. 1 muestra la proporción de países con datos representativos, por región de la OMS.

En la Región de África, los estudios examinados representan sobre todo a África Oriental, Occidental y Meridional; los países centrales están mal representados.

En la Región de las Américas estuvieron mejor representadas las naciones más pequeñas, por ejemplo, los países del Caribe, presuntamente porque la complejidad y el costo de obtener una estimación nacional son menores que en las naciones con grandes

FIGURA 1. Proporción de países con datos representativos sobre la caries dental a los 12 años de edad, por Región de la OMS

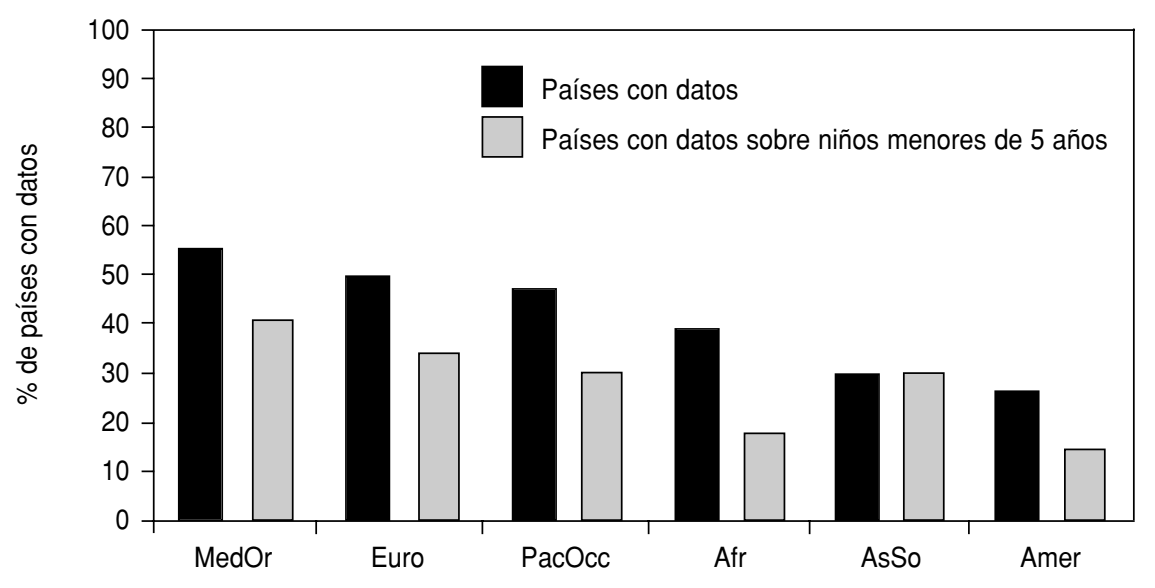


poblaciones, independientemente del producto nacional bruto (PNB).

La Región del Mediterráneo Oriental aportó la máxima proporción de conjuntos de datos nacionales (55\%), de los cuales $75 \%$ se recolectaron en el período 1991-1996 y 42\% provinieron de encuestas exploratorias.

En la Región de Europa hubo un total de 35 encuestas nacionales exploratorias o de conjuntos de datos de las juntas nacionales de salud. Nueve países de Europa Oriental tenían conjuntos de datos nacionales, de los cuales $89 \%$ se recopilaron con la metodología exploratoria. Casi toda la población del norte de Europa está sujeta a registro médico nacional; por consiguiente, la mayoría de los países del norte de Europa están representados en los datos recientes del BMDSB. La situación en otras partes de Europa es más complicada. No hay datos recientes sobre Italia y los que existen sobre la antigua Yugoslavia y Turquía corresponden a 1986 y 1987, respectivamente. En Europa Occidental se pueden hacer estimaciones razonables de la prevalencia de caries dental en niños de 12 años por causa de estudios bien diseñados en Austria, Bélgica, Francia y Suiza. No se dispuso de datos nacionales para el período de estudio en Alemania, Luxemburgo ni los Países Bajos.

La Región de Asia Sudoriental tiene el menor número de Estados Miembros de todas las regiones de la OMS por un amplio margen y, por ende, el total de tres conjuntos de datos de esa Región no es más escaso, en términos porcentuales, que los nueve conjuntos de la Región de las Américas. Sin embargo, el motivo de mayor preocupación es la falta de datos de los países más grandes, como la India - según las proyecciones, será el país más poblado del mundo- Indonesia y Bangladesh.

En la Región del Pacífico Occidental se ha dispuesto desde 1987 de datos nacionales sobre China obtenidos con una metodología de muestreo aleatorio. El Japón ha realizado encuestas nacionales de salud bucodental cada 6 años, la última en 1993. Australia y Nueva Zelandia proporcionan estadísticas de salud bucodental con regularidad y emplean metodologías de muestreo aleatorias. Entre 1986 y 1996, Australia realizó cuatro estudios nacionales y Nueva Zelandia, dos. En cuanto a las naciones insu- lares de Melanesia, Micronesia y Polinesia, están cubiertas solamente Kiribati, Samoa, Tonga y Vanuatu.

\section{Prevalencia de caries dental a los 12 años de edad}

Sesenta $(75 \%)$ de los 80 países con datos nacionales tuvieron un índice promedio de $\leq 3,0$ dientes $\mathrm{CPO}$, meta de la OMS/Federación Dental Internacional (FDI) para el año 2000. Cuarenta y cuatro de un total de 55 países en desarrollo tuvieron un índice promedio de dientes CPO de $\leq 3,0(80 \%)$. Catorce $(82 \%)$ de las 17 economías de mercado desarrolladas habían logrado esa meta, lo que presenta un marcado contraste con solamente dos $(25 \%)$ de las ocho economías en transición.

El índice medio de dientes CPO en los datos de la Región de África (cuadro 1) varió de 3,1 en Madagascar, la única media superior a la meta mundial, a 0,3 en la República Unida de Tanzanía. Se pudo identificar a tres grupos de países. Los países insulares (Cabo Verde, Madagascar y Mauricio) tuvieron un índice medio de caries dental muy su-

CUADRO 1. Prevalencia de caries dental a los 12 años, Región de África de la OMS, 1986-1996

\begin{tabular}{|c|c|c|c|c|c|c|c|c|}
\hline País & Encuesta $^{a}$ & Año & $n$ & $\%$ afectado & $\mathrm{DCPO}^{\mathrm{b}}$ & $\mathrm{DC}^{\mathrm{C}}$ & $\mathrm{DP}^{\mathrm{d}}$ & $\mathrm{DO}^{\mathrm{e}}$ \\
\hline Argelia & $\mathrm{R}$ & 1987 & 108 & $N D^{f}$ & 1,6 & 1,5 & 0,1 & 0,0 \\
\hline Benin & $\mathrm{NE}$ & 1994 & 1001 & 33 & 0,7 & 0,7 & 0,0 & 0,0 \\
\hline Burkina Faso & $\mathrm{R}$ & 1993 & 300 & 50 & 1,7 & 1,7 & 0,0 & 0,0 \\
\hline Cabo Verde & NA & 1989 & 282 & 78 & 2,8 & 2,6 & 0,2 & 0,0 \\
\hline Ghana & $\mathrm{R}$ & 1989 & 1493 & 20 & 0,4 & 0,4 & 0,0 & 0,0 \\
\hline Lesotho & $\mathrm{R}$ & 1991 & 354 & 20 & 0,4 & 0,3 & 0,0 & 0,0 \\
\hline Madagascar & $\mathrm{NE}$ & 1992-93 & 1992 & 75 & 3,1 & 3,0 & 0,1 & 0,0 \\
\hline Malawi & $\mathrm{R}$ & 1988 & 359 & 35 & 0,8 & 0,8 & 0,0 & 0,0 \\
\hline Mauricio & $\mathrm{R}$ & 1990 & 338 & 78 & 2,8 & 1,2 & 0,3 & 1,3 \\
\hline Namibia & $\mathrm{NE}$ & 1991 & 758 & 44 & 1,2 & 0,9 & 0,2 & 0,1 \\
\hline Níger & $\mathrm{NE}$ & 1988 & 619 & 60 & 1,7 & 1,7 & 0,0 & 0,0 \\
\hline Nigeria & NE & 1994 & 447 & 30 & 0,7 & 0,6 & 0,0 & 0,0 \\
\hline Rwanda & $\mathrm{R}$ & 1986 & 428 & 53 & 1,4 & 1,3 & 0,0 & 0,0 \\
\hline Senegal & $\mathrm{NE}$ & 1994 & 300 & 53 & 1,2 & 1,1 & 0,1 & 0,0 \\
\hline Sierra Leona & $\mathrm{R}$ & 1986 & 202 & 56 & 1,3 & 1,3 & 0,0 & 0,0 \\
\hline Sudáfrica & NA & 1988-89 & 5252 & 56 & 1,8 & ND & ND & ND \\
\hline República Unida de Tanzanía & $\mathrm{R}$ & 1994 & 1114 & ND & 0,3 & 0,3 & 0,0 & 0,0 \\
\hline Zimbabwe & NE & 1986 & 1008 & 47 & 1,2 & 1,1 & 0,0 & 0,1 \\
\hline
\end{tabular}

a $\mathrm{NA}=$ datos nacionales obtenidos con muestreo aleatorio; $\mathrm{NE}=$ estudio nacional exploratorio; $\mathrm{R}=$ datos subnacionales que permiten hacer una estimación nacional.

b $\mathrm{DCPO}=$ dientes cariados, perdidos y obturados.

${ }^{c} \mathrm{DC}=$ dientes cariados.

${ }^{\mathrm{d}} \mathrm{DP}=$ dientes perdidos.

e $\mathrm{DO}=$ dientes obturados.

${ }^{\dagger} \mathrm{ND}=$ No está disponible. 
CUADRO 2. Prevalencia de caries dental a los 12 años, Región de las Américas de la OMS, 1986-1996

\begin{tabular}{|c|c|c|c|c|c|c|c|c|}
\hline País & Encuesta $^{a}$ & Año & $n$ & $\%$ afectado & $\mathrm{DCPO}^{\mathrm{b}}$ & $\mathrm{DC}^{\mathrm{C}}$ & $\mathrm{DP}^{\mathrm{d}}$ & $\mathrm{DO}^{\mathrm{e}}$ \\
\hline Antigua & $\mathrm{R}$ & 1988-1989 & 400 & 38 & 0,7 & 0,5 & 0,1 & 0,1 \\
\hline Brasil & $\mathrm{NE}$ & 1993 & 3407 & $\mathrm{ND}^{f}$ & 4,9 & ND & ND & DN \\
\hline Dominica & NA & 1989 & 442 & 69 & 2,5 & 2,1 & 0,2 & 0,2 \\
\hline El Salvador & NE & 1989 & 857 & 90 & 5,1 & 4,7 & 0,2 & 0,2 \\
\hline Granada & NA & 1991 & 346 & 90 & 5,5 & 5,1 & 0,2 & 0,2 \\
\hline Guyana & NA & 1995 & 547 & 55 & 1,3 & ND & ND & ND \\
\hline San Vicente y las Granadinas & NA & 1991 & 181 & 82 & 3,2 & ND & ND & ND \\
\hline Uruguay & NA & 1992 & ND & ND & 4,2 & ND & ND & ND \\
\hline Estados Unidos de América & $\mathrm{NE}$ & $1986-1987$ & 3186 & 58 & 1,8 & ND & ND & ND \\
\hline
\end{tabular}

a-f Véanse las notas de pie de página correspondientes en el cuadro 1.

CUADRO 3. Prevalencia de caries dental a los 12 años, Región del Mediterráneo Oriental de la OMS, 1986-1996

\begin{tabular}{|c|c|c|c|c|c|c|c|c|}
\hline País & Encuesta $^{a}$ & Año & $n$ & $\%$ afectado & DCPO & $\mathrm{DC}^{\mathrm{C}}$ & $\mathrm{DP}^{\mathrm{d}}$ & $\mathrm{DO}^{\mathrm{e}}$ \\
\hline Chipre & NE & 1992 & 491 & 67 & 2,1 & 1,0 & 0,1 & 1,0 \\
\hline Egiptog & NA & 1991 & 4875 & 48 & 1,2 & 1,0 & 0,1 & 0,1 \\
\hline Jordania & $\mathrm{R}$ & 1995 & 280 & 90 & 3,3 & ND & ND & 0,1 \\
\hline Líbano & NA & 1993 & 648 & 93 & 5,7 & 5,1 & 0,2 & 0,3 \\
\hline Marruecos & NA & 1991 & 750 & 77 & 2,3 & 2,2 & 0,1 & 0,0 \\
\hline Túnez & NE & 1994 & 600 & 48 & 1,3 & 1,2 & 0,1 & 0,0 \\
\hline Emiratos Árabes Unidos & NA & 1995 & 17521 & ND & 1,6 & 1,1 & 0,0 & 0,5 \\
\hline Yemen & NE & 1987 & 550 & 79 & 3,1 & 3,0 & 0,0 & 0,1 \\
\hline
\end{tabular}

a-f Véanse las notas de pie de página correspondientes en el cuadro 1.

g Los datos corresponden a niños de 12 a 15 años.

perior al promedio; Argelia, Burkina Faso, el Níger y Sudáfrica tuvieron índices de caries dental un poco superiores al promedio; y los demás países registraron índices medios de dientes CPO de 0,3 a 1,4. Según la clasificación de la OMS del índice medio de dientes CPO a los 12 años de edad, seis de esos países estaban en la categoría de índice muy bajo $(0,0-1,1)$, nueve en la de índice bajo $(12-2,6)$ y tres en la de índice moderado $(2,7-4,4)$. Como era de esperarse, el componente de dientes obturados fue bajo en los países de África.

El cuadro 2 muestra los datos de prevalencia de caries dental en la Región de las Américas de la OMS. En la región del Caribe hubo una marcada diferencia en los índices de dientes $\mathrm{CPO}$, que variaron de 5,5 (Granada) a 0,7 (Antigua), así como en la proporción de niños afectados, que osciló entre 90\% (El Salvador, Granada) y
$38 \%$ (Antigua). Solamente en cuatro casos se situó el índice medio de dientes CPO en <3,0.

En la Región del Mediterráneo Oriental (cuadro 3), siete de los 12 países con un índice medio de 12 dientes CPO se agrupan alrededor de un valor mediano de 1,6. Los extremos fueron Djibouti y el Líbano con 0,4 y 5,7 dientes CPO, respectivamente $(93 \%$ de los niños de 12 años estaban afectados). Ocho de los Estados Miembros registraron un índice de caries bajo (de 1,2 a 2,6 dientes CPO). En conjunto, 75\% de los países tuvieron un índice medio de $\leq 3,0$ dientes CPO y solo Jordania, el Líbano y el Yemen notificaron uno de >3,0 dientes CPO, que guardó relación con los bajos porcentajes de niños exentos de caries (7-21\%).

En la Región de Europa (cuadro 4), todos los países con datos nacionales acopiados después de 1990 tuvieron un índice de $\leq 3,0$ dientes $\mathrm{CPO}$, con excepción de Hungría, Letonia, Lituania, Rumania y la Federación Rusa. Todos los países nórdicos indicaron índices de caries dental bajos, que variaron de 1,2 a 2,3 La mediana observada en todos los países europeos fue de 2,7. Los datos sobre el índice de dientes CPO en los países de Europa Occidental y Meridional fueron aproximadamente iguales a ese valor; Francia $(2,1)$, Suiza $(2,0)$ y el Reino Unido $(1,4)$ los tuvieron algo inferiores. Los países de Europa Oriental, con excepción de la antigua Yugoslavia (6,1 en 1986), Eslovenia $(2,6)$ y Uzbekistán $(1,3)$, tuvieron índices moderados de caries dental.

En la Región de Asia Sudoriental (cuadro 5), Nepal, Sri Lanka y Tailandia pertenecieron a la categoría de un bajo índice de dientes CPO $(1,2-2,6)$; el componente de dientes cariados fue grande. 
CUADRO 4. Prevalencia de caries dental a los 12 años, Región de Europa de la OMS, 1986-1996

\begin{tabular}{|c|c|c|c|c|c|c|c|c|}
\hline País & Encuesta ${ }^{a}$ & Año & $n$ & $\%$ afectado & $\mathrm{DCPO}^{\mathrm{b}}$ & $\mathrm{DC}^{\mathrm{C}}$ & $\mathrm{DP}^{\mathrm{d}}$ & $\mathrm{DO}^{\mathrm{e}}$ \\
\hline Austria & $\mathrm{RN}$ & 1994 & 491 & $N^{f}$ & 3,0 & ND & ND & ND \\
\hline Bélgica & $\mathrm{R}$ & 1991 & 3600 & 75 & 2,7 & 0,7 & 0,1 & 1,9 \\
\hline República Checa & $\mathrm{NE}$ & 1993 & 593 & 77 & 2,7 & 0,3 & 0,0 & 2,3 \\
\hline Dinamarca & $\mathrm{RN}$ & 1995 & 46504 & 50 & 1,2 & ND & ND & ND \\
\hline Finlandia & $\mathrm{RN}$ & 1991 & ND & 70 & 1,2 & ND & ND & ND \\
\hline Francia & $\mathrm{NA}$ & 1993 & 1331 & 65 & 2,1 & 0,6 & 0,2 & 1,2 \\
\hline Hungría & $\mathrm{NE}$ & 1991 & 898 & 90 & 4,3 & 2,0 & 0,3 & 1,9 \\
\hline Islandia & $\mathrm{NE}$ & 1996 & ND & ND & 1,5 & ND & ND & ND \\
\hline Israel & NE & 1990 & 762 & 79 & 3,0 & ND & ND & ND \\
\hline Letonia & $\mathrm{NE}$ & 1993 & 1180 & 86 & 5,8 & 3,5 & 0,2 & 2,0 \\
\hline Lituania & $\mathrm{NE}$ & 1994 & 1026 & 88 & 3,8 & ND & ND & ND \\
\hline Noruega & $\mathrm{RN}$ & 1991 & 48319 & 77 & 2,3 & 0,8 & 0,0 & 1,5 \\
\hline Polonia & $\mathrm{NE}$ & 1987 & 1380 & 90 & 4,4 & 2,7 & 0,1 & 1,6 \\
\hline Portugal & $\mathrm{NE}$ & 1989 & 704 & 89 & 3,2 & 2,2 & 0,3 & 0,7 \\
\hline Rumania & NE & 1992 & 392 & 91 & 4,0 & ND & ND & ND \\
\hline Federación Rusa & $\mathrm{R}$ & 1989-1995 & 5789 & 88 & 3,7 & 2,0 & 0,1 & 1,6 \\
\hline San Marino & NA & 1987 & 130 & 42 & 3,7 & 1,1 & 0,1 & 2,5 \\
\hline Eslovenia & NE & 1993 & 401 & 31 & 2,6 & 0,8 & 0,2 & 1,2 \\
\hline España & $\mathrm{NE}$ & 1993 & 511 & 68 & 2,3 & 1,3 & 0,1 & 0,9 \\
\hline Suecia & $\mathrm{RN}$ & 1994 & 72709 & 52 & 1,6 & ND & ND & ND \\
\hline Suiza & NA & 1987-1989 & 550 & 62 & 2,0 & 0,5 & 0,0 & 1,5 \\
\hline Turquía & $\mathrm{NE}$ & 1987 & 412 & 76 & 2,7 & 2,2 & 0,2 & 0,2 \\
\hline Reino Unido & NA & 1993 & 1499 & 53 & 1,4 & 0,4 & 0,1 & 0,8 \\
\hline Uzbekistán & NE & 1996 & 1143 & ND & 1,3 & 1,1 & 0,2 & 0,0 \\
\hline Antigua Yugoslavia & NE & 1986 & 301 & 99 & 6,1 & 3,8 & 0,5 & 1,8 \\
\hline
\end{tabular}

${ }^{a-f}$ Véanse las notas de pie de página correspondientes en el cuadro 1. $\mathrm{RN}$ = datos nacionales del registro nacional.

CUADRO 5. Prevalencia de caries dental a los 12 años, Región de Asia Sudoriental de la OMS, 1986-1996

\begin{tabular}{|c|c|c|c|c|c|c|c|c|}
\hline País & Encuesta $^{a}$ & Año & $n$ & $\%$ afectado & $\mathrm{DCPO}^{\mathrm{b}}$ & $\mathrm{DC}^{\mathrm{C}}$ & $\mathrm{DP}^{\mathrm{d}}$ & $\mathrm{DO}^{\mathrm{e}}$ \\
\hline Sri Lanka & $\mathrm{NE}$ & 1994-1995 & 2000 & 53 & 1,4 & 1,2 & 0,1 & 0,1 \\
\hline Tailandia & $\mathrm{NE}$ & 1994 & 2801 & 54 & 1,6 & 1,2 & 0,1 & 0,3 \\
\hline
\end{tabular}

a-f Véanse las notas de pie de página correspondientes en el cuadro 1.

En la Región del Pacífico Occidental (cuadro 6), el índice mediano de dientes $\mathrm{CPO}$ fue de 1,5 . Seis países, entre ellos China, se situaron en la categoría de índice muy bajo; cuatro en la de índice bajo; el Japón $(3,6)$ y la República de Corea $(3,0)$ tuvieron índices moderados de dientes CPO y Brunei Darussalam $(4,9)$ se situó en la categoría de índice alto.

\section{DISCUSIÓN}

Para efectos de la planificación y vigilancia de los programas de atención de salud bucodental, es indispensable tener un conjunto básico de esta- dísticas de referencia sobre el estado de la salud bucodental de la población en cuestión. La diversidad de los datos puede ser importante dentro del mismo país en relación con la zona (urbana, periurbana, rural) y las condiciones socioeconómicas. Si se contempla la posibilidad de hacer comparaciones internacionales, un método uniforme reviste particular importancia. Todos esos elementos justifican la función central que asigna la OMS al BMDSB.

Como parte de esa función, hace más de 20 años se tomó la iniciativa de establecer y emplear la metodología exploratoria de recolección de datos para facilitar la realización de encues- tas epidemiológicas periódicas y uniformes (6). En este sistema se emplea una metodología práctica de encuesta por medio de muestreo, que permite obtener información estadísticamente significativa y de importancia clínica para la planificación, a un costo mínimo. Este método también puede adaptarse a las necesidades de cualquier país, independientemente de su grado de desarrollo. Un objetivo importante es proporcionar datos comparables, que sean fidedignos pero no más precisos que las metas de la encuesta, de una comunidad a otra y con el transcurso del tiempo.

En los criterios para definir un estudio nacional se deben tener en cuenta 
CUADRO 6. Prevalencia de caries dental a los 12 años, Región del Pacífico Occidental de la OMS, 1986-1996

\begin{tabular}{|c|c|c|c|c|c|c|c|c|}
\hline País & Encuesta & Año & $n$ & $\%$ afectado & $\mathrm{DCPO}^{\mathrm{b}}$ & $\mathrm{DC}^{\mathrm{C}}$ & $\mathrm{DP}^{\mathrm{d}}$ & $\mathrm{DO}^{\mathrm{e}}$ \\
\hline Australia & NA & 1993 & 4780 & 44 & 1,1 & 0,4 & 0,0 & 0,7 \\
\hline Brunei Darussalam & NE & 1987 & 405 & 85 & 4,9 & 3,3 & 0,4 & 1,2 \\
\hline China & NA & 1987 & 26268 & $N D^{f}$ & 0,9 & ND & ND & ND \\
\hline Japón & NA & 1993 & 143 & 84 & 3,6 & 1,1 & 0,0 & 2,5 \\
\hline Kiribati & NE & 1992 & 108 & 28 & 0,7 & 0,5 & 0,1 & 0,1 \\
\hline República Democrática Popular Lao & NE & 1991 & 501 & 61 & 1,9 & 1,9 & 0,0 & 0,0 \\
\hline Nueva Zelandiag & NA & 1993 & 6577 & 50 & 1,5 & 0,0 & 0,0 & 1,5 \\
\hline República de Corea & NE & 1991 & 600 & 75 & 3,0 & 1,7 & 0,0 & 1,3 \\
\hline Samoa & NE & 1987 & 670 & ND & 2,5 & 2,4 & 0,0 & 0,1 \\
\hline Singapur & NA & 1995 & 449 & 42 & 1,0 & 0,0 & 0,0 & 1,0 \\
\hline Tonga & NE & 1986 & 1106 & 8 & 1,0 & 0,7 & 0,1 & 0,2 \\
\hline Vanuatu & NE & 1991 & 307 & ND & 1,1 & 1,0 & 0,0 & 0,0 \\
\hline Viet Nam & NE & 1990 & 300 & 58 & 1,8 & 1,7 & 0,0 & 0,1 \\
\hline
\end{tabular}

$\mathrm{a}^{-\mathrm{f}}$ Véanse las notas de pie de página correspondientes en el cuadro 1.

9 Los datos corresponden a niños de 12 a 13 años.

varios métodos de muestreo. Conviene examinar aquí la distinción básica entre la metodología de muestreo probabilístico aleatorio y el método exploratorio de la OMS, cuya representatividad se ha puesto en tela de juicio (3), pero también defendido (7), con la conclusión de que ofrece un grado de precisión satisfactorio sobre el estado de salud bucodental de una población para fines de planificación. Una encuesta nacional exploratoria debe incorporar suficientes sitios de examen para cubrir a todos los subgrupos importantes de la población que puedan tener diferentes tasas de morbilidad o factores de riesgo. Mientras que el método de muestreo probabilístico aleatorio trata de representar a toda la población al diseñar una muestra que reduzca al mínimo la posibilidad de pasar por alto cualquier variación, el método exploratorio define el número mínimo de grupos y conglomerados de edad que representarán amplias variaciones de importancia para los puntos incluidos en la encuesta con el fin de hacer una estimación nacional, con ponderación de los datos cuando sea necesario. La representatividad de cualquier muestra también está vinculada a su tamaño. El número recomendado de niños por grupo de edad para las encuestas exploratorias en lugares con una prevalencia moderada de caries dental es de 300, pero varía según la prevalencia de la caries (muy baja, baja, moderada, alta y muy alta) y el grado de precisión necesario. En 5\% de los estudios nacionales exploratorios incluidos en el BMDSB, el tamaño de la muestra fue de $<300$ sujetos.

A partir de la información del BMDSB, la OMS preparó anualmente hasta 1996 un documento sobre la caries dental a los 12 años de edad (8), en que se cita el índice de dientes CPO en cada uno de sus Estados Miembros y, de esa manera, proporciona información sobre los índices comparativos y tendencias generales de la caries dental. Se incluyen los resultados del estudio más representativo de cada Estado Miembro, sujetos a confirmación por las oficinas regionales de la OMS. Puesto que los registros también pueden incluir datos regionales o subnacionales que, según se estima, dan aproximaciones razonables de las medias nacionales, es importante consultar las referencias citadas al final del documento. De esa forma, es posible identificar las fuentes de datos y realizar investigaciones metodológicas para evaluar el origen nacional, departamental o local de la información notificada, por país y año.

La principal finalidad del presente documento, al presentar la mejor $\mathrm{y}$ más reciente estimación de los datos sobre el índice nacional medio de caries dental, es dar una idea general aproximada y promover las actividades tendientes a mejorar la informa- ción donde esté desactualizada o no represente el promedio nacional a cabalidad. Si se necesita un enfoque más riguroso, la única forma real de lograrlo es realizar un análisis detallado de los datos, como se describe en el presente artículo.

En fecha reciente se ha presentado la información acopiada sobre salud y enfermedades bucodentales en tres publicaciones (9-11). En 1996, la Organización Europea de Investigación sobre la Caries Dental (ORCA) publicó datos sobre la prevalencia y gravedad de la caries dental en 35 países europeos en el período 1983-1995 (9). Se cita un total de 21 acopios de datos nacionales en 11 países. En un informe sobre la situación de la salud bucodental en África, se presentaron datos sobre el índice de dientes CPO a los 12 años en el período 1980-1994 en 15 países (10). Sin embargo, no fue posible identificar las referencias ni clasificar los datos como regionales o nacionales y 13 de las encuestas nacionales efectuadas en la Región de África dejaron de citarse en el BMDSB. La OPS ha notificado los resultados de varias encuestas sobre la caries dental a los 12 años en 29 países (11); ninguno de esos datos se incluye en el BMDSB como cifra nacional. En un número especial del International Dental Journal de 1994 se presentaron datos sobre la prevalencia de caries dental en diferentes países. Las observaciones provenien- 
tes de Dinamarca (12), Finlandia (12), Suecia (12), Suiza (13) y el Reino Unido (14) son compatibles con los datos del BMDSB. Esos resultados también son compatibles con los descritos por Burt (15), cuyos datos se tomaron de estudios regionales o locales en Canadá y Estados Unidos de América, con excepción de los tomados del estudio nacional de 1986-1987 que se efectuó en el último país citado. Sin embargo, las observaciones de Fejerskov et al. (16) sobre la salud bucodental a los 12 años de edad en China y África se basaron en datos escasos. Esos autores analizaron la Región de África solamente por medio de estudios hechos en Nigeria, la República Unida de Tanzanía y Uganda, a pesar de haber datos nacionales de 13 países africanos en el BMDSB.

Veintiuno de los Estados Miembros de la OMS realizaron más de una encuesta nacional en el período 19861996, pero en 110 países no se efectuó ninguna. Esto indica claramente la carencia de datos recientes sobre muchos países y la desigualdad entre los diferentes países y regiones en lo que respecta a la disponibilidad de información sobre el estado de salud bucodental de las poblaciones. El problema fue más grave en la Región de Asia Sudoriental y la Región de las Américas; por otra parte, la proporción sorprendentemente baja $(50 \%)$ de datos nacionales en los Estados Miembros de la Región de Europa admite varias explicaciones. Primero, últimamente se ha hecho un esfuerzo por recolectar datos nacionales: $68 \%$ de los estudios nacionales reseñados en nuestro informe se realizaron en los últimos cinco años. Segundo, casi todos los países con datos nacionales los han producido con regularidad; por ejemplo, Francia ha efectuado tres estudios na- cionales en cinco años. Tercero, el número de países de Europa ha aumentado mucho desde la disolución de la Unión Soviética, de 34 países en 1990 a 53 hoy en día. Las estadísticas sobre la caries dental en los países de la antigua Unión Soviética fueron escasas; solo Letonia, Lituania, la Federación Rusa y Uzbekistán están representados en el banco de datos. Sin embargo, la serie de encuestas nacionales exploratorias que comenzó en 1985 en la Unión Soviética e incluyó siete regiones, cuyos resultados son publicados regularmente por el centro colaborador de la OMS en Moscú, ofrecen información muy importante sobre la gravedad de la situación de la salud bucodental en las economías en transición. En los países del norte de Europa, con excepción del Reino Unido, el problema está en el acceso a los datos recolectados, ya que no siempre figuran en las publicaciones disponibles. Por último, se debe recalcar que hay una asombrosa falta de datos recientes sobre la situación de los países más extensos del mundo. Por tanto, no se dispuso de datos nacionales actualizados sobre Bangladesh, Brasil, India e Indonesia, que figuran entre los 10 países más poblados del mundo.

Como era de esperarse, hubo enormes diferencias en el tamaño de la muestra según el tipo de encuesta y cultura, las estructuras administrativas y el estado previsto de la salud bucodental. En un total de 34\% de los estudios nacionales, el tamaño de la muestra fue de $>1000$ y en $13 \%$, de $<300$. Los países nórdicos proporcionaron amplios datos provenientes de estadísticas de servicio recopiladas por las juntas nacionales de salud, según informes que cubrieron a la mayoría de las personas en la cohorte de edad objeto de estudio. Los datos de esos países son estadísticas muy fidedignas porque provienen de sistemas integrales de servicios de salud bucodental para los niños en los que se basan. El tamaño mediano de la muestra en la Región de África fue de 438 sujetos. Por desgracia, no se pudieron incluir algunos estudios nacionales hechos recientemente en Benin (1995) y Zimbabwe (1996) porque el tamaño de la muestra de niños de 12 años era demasiado pequeño según los criterios del BMDSB. En la Región del Pacífico Occidental, en una encuesta nacional efectuada hace poco en el Japón, el tamaño de la muestra de niños de 12 años de edad (143 niños) fue también pequeño, pero debería ser aceptable por ser el resultado de un sistema de muestreo sumamente complejo para todas las edades que se ha empleado cada seis años desde 1957.

El BMDSB es actualmente el único instrumento de esa naturaleza que puede proporcionar información estandarizada sobre el estado de la salud bucodental de los niños; sin embargo, hasta cierto punto los investigadores se han olvidado de que existe. Se pueden solicitar estadísticas y resúmenes a la OMS y obtener en la World Wide Web (WWW) (http://www.whocollab. odont.lu.se). También es posible formular preguntas de investigación más específicas empleando el BMDSB; hasta la fecha este recurso se ha mantenido inexplorado en gran parte. La OMS aspira a promover el uso de este instrumento de trabajo y en un futuro próximo se propone actualizar mensualmente el BMDSB en la WWW. Eso permitiría que los usuarios enviaran comentarios rápidamente sobre la información disponible y facilitaría el traslado de datos epidemiológicos al BMDSB.

\section{REFERENCIAS}

1. Leclercq MH, Bames DE, Sardo Infirri J. Oral Health: Global trends and projections. World Health Stat Q 1987;40:116-120.

2. Leopold CT, Mander C, Utting C, Watkins $K$, Rock WP. The World Health Organization goals for oral health: A progress report. Community Dent Health 1991;8:245-251.
3. European Organization for Caries Research. Symposium report: Caries status in Europe and predictions of future trends. Caries Res 1990;24:381-396.

4. World Health Organization. Oral health surveys: Basic methods. 3rd ed. Geneva: WHO; 1994.
5. United Nations. World economic and social survey 1994. New York: United Nations; 1994.

6. Pakhomov GN. The World Health Organization's Oral Health Programme. FDI World 1996;5(3):22-23.

7. Bourgeois D, Leclercq MH, Barmes D. Evaluation of the World Health Organization 
pathfinder methodology for oral health surveys in industrialized countries. Community Dent Health 1992;9:381-384.

8. World Health Organization. DMFT levels at 12 years, 1996. Geneva: WHO; 1996.

9. European Organization for Caries Research. Symposium report: The prevalence of dental caries in Europe 1990-1995. ORCA Saturday Afternoon Symposium 1995. Caries Res 1996; 30:237-255.

10. Hobdell MH, Thorpe SJ. Oral health in Africa: Where are we now and why is there in- equality? En: Myburgh NG, ed. Proceedings of a CDA/WHO Workshop, Cape Town, South Africa, 1-3 April 1996. Cape Town: 1996. pp. 1-9.

11. Watson MR. Caries and periodontal diseases in Latin America and the Caribbean. Washington, DC: Pan American Health Organization; 1994. pp. 1-9.

12. Von der Fehr FR. Caries prevalence in the Nordic countries. Int Dent J 1994;44:371-378

13. Marthaler TM, Steiner M, Menghini G, Bandi A. Caries prevalence in Switzerland. Int Dental J 1994;44:393-341.
14. Downer MC. Caries prevalence in the United Kingdom. Int Dent J 1994;44:365-370.

15. Burt BA. Trends in caries prevalence in North American children. Int Dent J 1994;44: 403-413.

16. Fejerskov O, Baelum V, Luam WM, Manji F. Caries prevalence in Africa and the People's Republic of China. Int Dent J 1994;44 425-433.

ABSTRACT The global oral health situation of 12-year-old children-decayed, missing, filled teeth (DMFT) index and the percentage of population affected-is described in this article using the latest representative studies for 80 countries included in the WHO Global

WHO Global Data Bank, Oral Data Bank (GODB) between 1986 and 1996.

1986-96: An overview of

The quantity of information varied considerably: $68 \%$ of developed market econooral health surveys at 12 years of age mies had at least one national data set, compared with $38 \%$ of developing countries and $36 \%$ of economies in transition. By WHO region, the proportions were as follows: Eastern Mediterranean, 55\%; European, 50\%; Western Pacific, 48\%; African, 39\%; South-East Asia, 30\%; and the Americas, 26\%.

Globally, the weighted DMFT index for all data in the GODB is $<3.0 \%$, the WHO/ Fédération Dentaire Internationale goal for the year 2000. For the data reviewed in this article, achievement and nonachievement of this goal are discussed, as is the variation in DMFT means and proportions of children affected for various country groupings. There are difficulties in obtaining recent data for many countries, but the article emphasizes the need to maintain and develop the GODB to facilitate the compilation of valid, reliable and comparable data on oral health. 